Original article of Polymer Bulletin

\title{
Hydrolysis of Polyurea under High Pressure of Carbon Dioxide
}

Suguru Motokucho, ${ }^{1}$ Takeshi Matsumoto, ${ }^{1}$ Yu Nakayama, ${ }^{1}$ Ryohei Horiuchi, ${ }^{1}$ Hiroshi Morikawa $^{2}$ and Hisayuki Nakatani ${ }^{1}$

${ }^{1}$ Chemistry and Materials Engineering Program school of Engineering, Nagasaki University, 1-14, Bunkyo-Machi, Nagasaki-shi, Nagasaki 852-8521,JAPAN

${ }^{2}$ Department of Applied Chemistry, Kanagawa Institute of Technology, 1030, Shimo-ogino, Atsugi, Kanagawa 243-0292,JAPAN

Correspondence to: Suguru Motokucho (E-mail: motoku@nagasaki-u.ac.jp) 


\begin{abstract}
A chemical decomposition of polyurea (PUA) by hydrolysis under high pressure of carbon dioxide $\left(\mathrm{CO}_{2}\right)$ was proposed. The hydrolysis of PUA was carried out at $190{ }^{\circ} \mathrm{C}$ for $2 \mathrm{~h}$ under $7.0 \mathrm{MPa}$ of $\mathrm{CO}_{2}$ in the presence of water. The hydrolysis reaction gave white residual solid and water soluble compound. The white residual solid was characterized to be degraded PUA by FT-IR spectrum and elemental analysis.

${ }^{1} \mathrm{H}-\mathrm{NMR}$ spectra of the water soluble compound revealed that the hydrolysis of PUA produced diamine only from repeating unit of PUA, which was a component of PUA. This hydrolysis gave the corresponding diamine for quantitative yields.
\end{abstract}

\title{
Key words
}

Hydrolysis reaction, Carbonic acid, Carbon dioxide, Sustainable chemistry 


\section{INTRODUCTION}

Efficient use of resources and recycling of products are indispensable for establishing a sustainable society. Chemical recycling involving decomposition of polymers to their respective monomers and reproduction of the polymers from the obtained monomers is the most desirable way for sustainable chemistry.[1-3] Although there are several key challenges for its application such as decomposing the polymers to monomers, they should require high selectivity of conversion to desired monomers and easy techniques utilizing nonhazardous chemicals for the decomposition. Several chemical recycling processes are commercialized, i.e., solvolysis methods,[4-9] in which organic solvents and alkali catalysts are used; however, the development of much safer and environmentally benign systems is desired. High-temperature liquid or supercritical water has attracted much attention as an alternative to a harmful organic solvent because of its high proton concentration, which enhances the rates of acid-catalyzed reactions such as hydrolysis in water without adding any hazardous acids.[10-12]

Campanelli et al. reported that high-temperature water at $250{ }^{\circ} \mathrm{C}$ was effective for hydrolysis of Poly(ethylene terephthalate) (PET) to terephthalic acid (TPA) and ethylene glycol (EG).[13] Sato et. al. reported that PET was depolymerized to TPA and EG in water at higher than $220{ }^{\circ} \mathrm{C}$ and the hydrolysis of PET was enhanced by the addition of TPA in the reaction system.[14] They described that protons effectively catalyzed hydrolysis of PET by the addition of TPA. The addition of TPA serves protons for the hydrolysis of PET. However, for the polymer which consists of the monomer of non-acidity, such as polyurea (PUA), the degradation of polymer cannot be enhanced by a monomer addition. PUA is produced as by-product, when an 
isocyanate was produced from the reaction of diamine with phosgene in industrially. Generally, the produced PUA is incinerated and produce $\mathrm{CO}_{2}$ and NOx. These gases are environmentally harmful chemicals. From the point of protection of our environment, the development of hydrolysis reaction of PUA is important.[9, 15] Here we investigate the hydrolysis of PUA by the using water under high pressure of $\mathrm{CO}_{2}$. It is well-known that the reversible reaction of $\mathrm{CO}_{2}$ with $\mathrm{H}_{2} \mathrm{O}$ forms carbonic acid, leading to a temporary generated proton specie in situ with $\mathrm{p} K_{\mathrm{a}}$ value as low as 3.6.[16,17] The $\mathrm{p} K_{\mathrm{a}}$ of carbonic acid is similar $\mathrm{p} K_{\mathrm{a}}$ of TPA. The formed carbonic acid,[17] which is in situ Brønsted acid, could serve as an acid catalyst. We have already patented the hydrolysis of PUAs.[15] However, details of the hydrolysis condition and reaction mechanism were unclear, and studied in this literature.

\section{EXPERIMENTAL}

Materials. $\quad$ 1,6-Hexamethylene diisocyanate (HDI, Nippon Polyurethane Industry Co. Ltd., Yamaguchi, Japan), toluene diisocyanate (TDI, WAKO Chemicals Co., Ltd., Osaka, Japan) and $N, N$-dimethyl formamide (DMF, WAKO Chemicals Co., Ltd., Osaka, Japan) were used after distillation under reduced pressure. 4,4'-Diphenylmethane diisocyanate (MDI, Nippon Polyurethane Industry Co. Ltd., Yamaguchi, Japan) was used as received.

Characterization. IR spectrum was recorded with Bio-Rad Laboratories FTS $3000 \mathrm{MXN}$, and the values are given in $\mathrm{cm}^{-1}$. ${ }^{1} \mathrm{H}$-nuclear magnetic resonance $\left({ }^{1} \mathrm{H}-\mathrm{NMR}\right)$ spectra of products were recorded on a JNM-GX400 at $400 \mathrm{MHz}$. Elemental analyses of samples were analyzed on PerkinElmer PE2400II. 
Preparation of PUA. To a solution of HDI (8.40 g, $500 \mathrm{mmol})$ in DMF (150 mL), water $(0.90 \mathrm{~g}, 500 \mathrm{mmol})$ was added at room temperature, and the reaction temperature was raised up to $80{ }^{\circ} \mathrm{C}$. Then the white solid was precipitated in the reaction mixture, and the mixture was stirred for $24 \mathrm{~h}$. The resulting precipitate was collected by filtration and dried in vacuo to give a white solid of PUA-HDI (6.95 g, 98\%). PUA-HDI was insoluble in several solvents, such as DMF, dimethyl sulfoxide (DMSO), methanol, ethanol, toluene, chloroform, hexane, ethyl acetate and water.

IR $(\mathrm{KBr}) 3330(\mathrm{v}(\mathrm{NH})), 2935,2857(\mathrm{v}(\mathrm{C}-\mathrm{H})), 1618(\mathrm{v}(\mathrm{C}=\mathrm{O}))$ and $1575(\delta(\mathrm{N}-\mathrm{H})) \mathrm{cm}^{-1}$; Elemental Analysis, Calcd. for $\mathrm{C}_{7} \mathrm{H}_{14} \mathrm{~N}_{2} \mathrm{O}: \mathrm{C}, 59.12 \% ; \mathrm{H}, 9.92 \% ; \mathrm{N}, 19.70 \%$. Found: C, 59.09\%; H, 10.11\%; N, 19.80\%.

Preparation of PUA-MDI and PUA-TDI: The preparation of PUA-MDI and PUA-TDI were follow a similar procedure as described above. The yields of PUA-MDI and PUA-TDI were 99\% and 98\%, respectively. These PUAs were white solid and insoluble in several solvents, such as DMF, DMSO, methanol, ethanol, toluene, chloroform, hexane, ethyl acetate and water.

PUA-MDI: IR (KBr) 3306(v(NH)), 3033(v(C-H)), 2899(v(C-H)), 1643(v(C=O)) and 1595(v $(\mathrm{C}=\mathrm{C})$ aromatic ring $), 1304(\delta(\mathrm{C}-\mathrm{N})) \mathrm{cm}^{-1}$; Elemental Analysis, Calcd. for $\mathrm{C}_{14} \mathrm{H}_{12} \mathrm{~N}_{2} \mathrm{O}: \mathrm{C}, 74.98 \% ; \mathrm{H}, 5.39 \% ; \mathrm{N}, 12.49 \%$. Found: C, 75.12\%; H, 5.21\%; N, $13.80 \%$.

PUA-TDI: IR (KBr) 3301(v(NH)), 2924, 2861(v(C-H)), 1645(v(C=O)), $1542(\delta(\mathrm{N}-\mathrm{H}))$ and $1228 \mathrm{~cm}^{-1}$; Elemental Analysis, Calcd. for $\mathrm{C}_{8} \mathrm{H}_{8} \mathrm{~N}_{2} \mathrm{O}: \mathrm{C}, 64.85 \%$; H, 5.44\%; N, 18.91\%. Found: C, 64.71\%; H, 5.56\%; N, 19.04\%. 
General procedure of hydrolysis of PUA. $0.5 \mathrm{~g}$ of PUA-HDI and $20 \mathrm{~mL}$ of water were placed into a $200 \mathrm{~mL}$ stainless autoclave equipped with a pressure gauge followed by the introduction of appropriate amount of liquid $\mathrm{CO}_{2}$. Then, the autoclave was heated using a band heater under high pressure of $\mathrm{CO}_{2}(7.0 \mathrm{MPa})$. After a given reaction time, the reactor was rapidly immersed into an ice bath to cool down to room temperature. Finally, the reaction mixture was collected from the reactor and separated to the filtrate and residual solid fractions with a filter paper. The obtained solid was dried under vacuum and weighed. The solid was analyzed by FT-IR spectroscopy and elemental analysis. The filtrate was concentrated by the rotary evaporator and gave the non-volatile residue. The non-volatile residue was weighed after drying. The residue was analyzed by ${ }^{1} \mathrm{H}-\mathrm{NMR}$ spectroscopy and elemental analysis. The residue was purified by silica-gel column chromatography (eluent with $\mathrm{CHCl}_{3}$ ).

\section{RESULTS and DISCUSSION}

\section{Preparation of PUAs}

Three PUAs were easily obtained by the addition reaction of several diisocyanates with water. The equal molar reaction completed within $24 \mathrm{~h}$ at $80{ }^{\circ} \mathrm{C}$. All PUAs did not dissolve in the water and various organic solvents, such as DMF, DMSO, methanol, ethanol, toluene, chloroform, hexane and ethyl acetate. The PUAs were characterized by Fourier transform infrared (FT-IR) spectroscopy and elemental analysis.

\section{Hydrolysis Reaction of PUA-HDI and Identification of Reaction Mixture}


The hydrolysis of PUA-HDI (0.50 g) was carried out under high pressure $\mathrm{CO}_{2}$ of $7.0 \mathrm{MPa}$ at $190{ }^{\circ} \mathrm{C}$ for $0.5 \mathrm{~h}$. The reaction mixture was filtrated and gave residual solid and filtrate. At the first, the residual solid was washed with diluted water and methanol and dried in vacuo at room temperature for $24 \mathrm{~h} . \quad 0.23 \mathrm{~g}$ of the white solid was obtained at this reaction condition. The absorption bands of FT-IR spectrum of residue were observed identical to PUA-HDI's ones. This result suggested that the terminal amino group affected the nitrogen content. These experimental data suggested that the residue have same repeating structure of PUA-HDI and was converted to low molecular weight of PUA-HDI via the hydrolysis reaction.

Next, the filtrate was concentrated by the rotary evaporator and gave $0.30 \mathrm{~g}$ of residual white solid compound as a water soluble compound. Its IR absorption at 3340, 2930, 2860, 1640 and $1611 \mathrm{~cm}^{-1}$ and result of elemental analysis suggested the preparation of carbamic acid salt of 1,6-hexamethylene diamine (HDA) from the hydrolysis reaction of PUA-HDI. The water soluble compound was also analyzed with the ${ }^{1} \mathrm{H}-\mathrm{NMR}$ spectroscopy in $\mathrm{D}_{2} \mathrm{O}$. Figure 1 shows ${ }^{1} \mathrm{H}-\mathrm{NMR}$ spectrum of water soluble compound. Observation of resonance peaks at $1.34(\mathrm{~m}, 4 \mathrm{H}), 1.58(\mathrm{~m}, 4 \mathrm{H})$ and 2.85-2.92 $(\mathrm{m}, 4 \mathrm{H})$, assignable to protons of methylene groups of 1,6-, 2,5-, 3,4-positions, respectively, on ${ }^{1} \mathrm{H}-\mathrm{NMR}$ spectrum indicated formation of HDA, which was from corresponding carbamic acid salt aforementioned.

The yield of HDA was estimated from following equation:

Weight of water soluble compound from filtrate $(\mathrm{g})=\mathbf{A}$

Molecular weight of carbamic acid of HDA $=160.21$ 
Weight of obtained residual solid after hydrolysis reaciton $(\mathrm{g})=\mathbf{B}$

Molecular weight of repeating unit of PUA-HDI $=142.20$

$$
\text { Yield of HDA }=\frac{\mathbf{A} / 160.21}{[0.50(\mathrm{~g})-\mathbf{B}] / 142.20} \times 100
$$

\section{Effect of the Reaction Conditions for Hydrolysis Reaction of PUAs Reaction Time and $\mathrm{CO}_{2}$}

The hydrolysis reaction of PUA-HDI was carried out at $190^{\circ} \mathrm{C}$ under $7.0 \mathrm{MPa}$ of $\mathrm{N}_{2}$ or 7.0 MPa of $\mathrm{CO}_{2}$ as shown in Figure 2. At 7.0 MPa of $\mathrm{N}_{2}$, yield of HDA did not reach $20 \%$ even after $2 \mathrm{~h}$. Under high pressure of $\mathrm{N}_{2}$, the hydrothermal decomposition of PUA occurred. On the other hand, under 7.0 $\mathrm{MPa}$ of $\mathrm{CO}_{2}$, when the reaction time was elongated from 0.5 to $2.0 \mathrm{~h}$, the yield of HDA was smoothly increased from $52 \%$ to quantitative yield. It was concluded that the hydrolysis of PUA-HDI was accelerated under high pressure of $\mathrm{CO}_{2}$.

\section{Temperature and other PUA}

This hydrolysis reaction was applied to each temperature to investigate the effect of the reaction temperature on this hydrolysis reaction. Figure 3 shows the relationship between temperature and yield of $\mathrm{HDA}$ for $2 \mathrm{~h}$ under $7.0 \mathrm{MPa}$ of $\mathrm{CO}_{2}$. At $120{ }^{\circ} \mathrm{C}$, the yield of HDA was $6.1 \%$. The yield of HDA was increased with increasing temperature. We confirmed that the hydrolysis reaction of PUA was occurred over $120{ }^{\circ} \mathrm{C}$, and accelerated under high pressure $\mathrm{CO}_{2}$. Then, MDI-PUA and TDI-PUA as aromatic PUAs were submitted for the hydrolysis reaction under high pressure $\mathrm{CO}_{2}$. MDI-PUA and TDI-PUA were hydrolyzed to the corresponding diamines (DAs), 
4,4'-diaminodiphenyl methane and diamino toluene, in excellent yields, respectively. These indicate that this hydrolysis reaction can be applied to various polyurea.

\section{Pressure of $\mathrm{CO}_{2}$}

Figure 4 shows the relationship between pressure of $\mathrm{CO}_{2}(2.0-14.0 \mathrm{MPa})$ and yield of corresponding DAs at 160 and $190{ }^{\circ} \mathrm{C}$ for $2 \mathrm{~h}$. At $190{ }^{\circ} \mathrm{C}$, all hydrolyses of PUAs were shown in similar trends. The yield of DA was increased with increasing pressure. It reached quantitative one at $190{ }^{\circ} \mathrm{C}$. Therefore, the effect of $\mathrm{CO}_{2}$ pressure was not clear at $190{ }^{\circ} \mathrm{C}$. On the other hand, at $160{ }^{\circ} \mathrm{C}$, it reached maximum yield at 7.0 $\mathrm{MPa}$, which was below critical pressure of $\mathrm{CO}_{2}$ at the reaction temperature. Especially, TDA from TDI-PUA was produced with high yields at the $160{ }^{\circ} \mathrm{C}$. This result might suggest that the crystallinity or hydrogen-bonding ability of PUA affect hydrolysis reaction. This speculation was suggested from the compared with MDI-PUA. The increasing of yield of DA until ca. $7 \mathrm{MPa}$ might be from the increased formation of carbonic acid due to the dependent on $\mathrm{CO}_{2}$ pressure. On the other hand, the opposing behavior over ca. $7 \mathrm{MPa}$ might come from the decreased dissociation and/or transfer of protons from carbonic acid due to the decreased polarity of the solvent. $[18,19]$

In order to confirm effect of carbonic acid, we investigated the effect of the amount of water on yield of HDA. This reaction reached quantitative yield of HDA at relatively higher temperature of $190{ }^{\circ} \mathrm{C}$, meaning that the effect of the water is not obvious at $190^{\circ} \mathrm{C}$. Therefore we carried out the reaction at not $190{ }^{\circ} \mathrm{C}$ but $160^{\circ} \mathrm{C}$. Figure 5 shows that the relationship between amount of water and yield of HDA at $160{ }^{\circ} \mathrm{C}$ for $2 \mathrm{~h}$ under 7.0 $\mathrm{MPa}$ of $\mathrm{CO}_{2}$ pressure. The yields of HDA increased slightly 
from 10 to $40 \mathrm{~mL}$ of water. Over $40 \mathrm{~mL}$ of water, the yield of HDA decreased with increasing amount of water. It is assumed that the dissociation of protons from carbonic acid increased due to increasing the polarity of solution by addition of $10 \sim 40$ $\mathrm{mL}$ water. It is also assumed that the concentration of carbonic acid was diluted by addition of excess amount of $40 \sim 80 \mathrm{~mL}$ water. This result means concentration of carbonic acid and dissociation of protons from the acid are important factors for the hydrolysis reaction of PUA. Indeed, this assumption was well consistent with that from the relationship between $\mathrm{CO}_{2}$ pressure and yields of DAs in Figure 4.

\section{Mechanism of Hydrolysis Reaction}

Figure 6 illustrates a plausible mechanism for this hydrolysis of PUA. First, carbonic acid was formed by the reaction of $\mathrm{CO}_{2}$ with $\mathrm{H}_{2} \mathrm{O}$. The acid could release protons by the dissociation of in situ reaction. Then the protons activated to carbonyl group of urea one. And the activated urea group reacted with $\mathrm{H}_{2} \mathrm{O}$ to form oxonium cationic intermediate. The intermediate released proton and gave hemiacetal groups. Immediately, the hemiacetal groups gave carbamic acid group and amino ones.[20] The carbamic acid group gave amino group with releasing $\mathrm{CO}_{2}$.

\section{CONCLUSION}

We have successfully demonstrated the efficient promotion of hydrolysis of PUAs in high-temperature water under high pressure of $\mathrm{CO}_{2}$ which are nonhazardous in nature. Moreover another important advantage of this process is that it does not require neutralization and further purification of the corresponding DA obtained from PUA. The advantageous feature is not attained by various acids. 
Although further investigation is needed to precisely settle the mechanism, we have provided a green and general method for the hydrolysis of urea linkage via carbonic acid from the reaction of $\mathrm{CO}_{2}$ with $\mathrm{H}_{2} \mathrm{O}$.

\section{Acknowledgments}

This work was supported by the Ministry of Education, Culture, Sports, Science and Technology KAKENHI Grant-in-Aid for Young Scientists (B) Grant Number 25810078 and Japan Society for the Promotion of Science is funding agency of Japanese Ministry of Science Grant-in-Aid for "Program for Advancing Strategic International Networks to Accelerate the Circulation of Talented Researchers". And the author is sincerely grateful to Dr. Toshio Inoue, JX Nippon Oil \& Energy Corporation. 


\section{REFERENCES AND NOTES}

1. D. Paszun, T. Spychaj, Ind. Eng. Chem. Res., 36, 1373, (1997).

2. S. D. Mancini, M. Zanin, J. Appl. Polym. Sci., 76, 266, (2000).

3. D. K. Schneiderman, M. E. Vanderlaan, A. M. Mannion, T. R. `anthani, D. C. Batiste, J. Z. Wang, F. S. Bates, C. W. Macosko, and M. A. Hillmyer, ACS Macro Letters, 5, 515-518, (2016).

4. EI du Pont de Nemours Co., Pat. GB 1955001104219550418, (1957).

5. K. Sato, K. Sumitani, Jpn. Pat. 7,309,810, (1995).

6. K. Ishihara, K. Ishida, Jpn. Pat. 11,302,227, (1999).

7. Y. Saitou, M. Nakajima, Koubunshi, 54, 129, (2005).

8. K. Sato, Materiaru Raifu Gakkaishi, 16, 7, (2004).

9. X. Lu, Z. Cui, W. Wei, J. Xie, L. Jiang, J. Huang, J. Liu, Chem. Eng. J., 284(15), 478-4686, (2016).

10. Y. Nagase, The review of high pressure science and technology, 12, 217, (2002).

11. F. L. P. DeResende, M. E. Neff, P. E. Savage, Energy \& Fuels, 21, 3637 (2007).

12. S. E. Hunter, C. E. Ehrenberger, and P. E. Savage, J. Org. Chem, 71, 6229, (2006).

13. J. R. Campanelli, D. G. Cooper, M. R. Kamal, J. Appl. Polym. Sci., 53, 985, (1994).

14. E. Sato, M. Mine, N. Osada, A. Hiyoshi, K. Yamaguchi, K. Bando, Y. Masuda, and M. Shirai, Chem. Lett., 38, 3, 268, (2009).

15. M. Furukawa, K. Kojio, S. Motokucho, PCT patent application WO 2010023922.

16. M. J. Welch, J. F. Lifton, J. A. Seck, J. Physical Chemistry, 73, 3351, (1969).

17. K. N. West, C. Wheeler, J. P. McCarney, K. N. Griffith, D. Bush, C. L. Liotta, and C. A. Eckert, J. Phys. Chem. A, 105, 3947, (2001).

18. X. Xie, C. L. Liotta, and C. A. Eckert, Ind. Eng. Chem. Res., 43, 2605, (2004). 
19. R. Ma, A.-H. Liu, C.-B. Huang, X.-D. Li and L.-N. He, Green Chem. 15, 1274-1279, (2013).

20. S. Motokucho, S. Mukai, M, Furukawa, K. Kojio, K. Yoshinaga, Nippon Gomu Kyokaishi 85, 5, 157, (2012). 
Figure 1

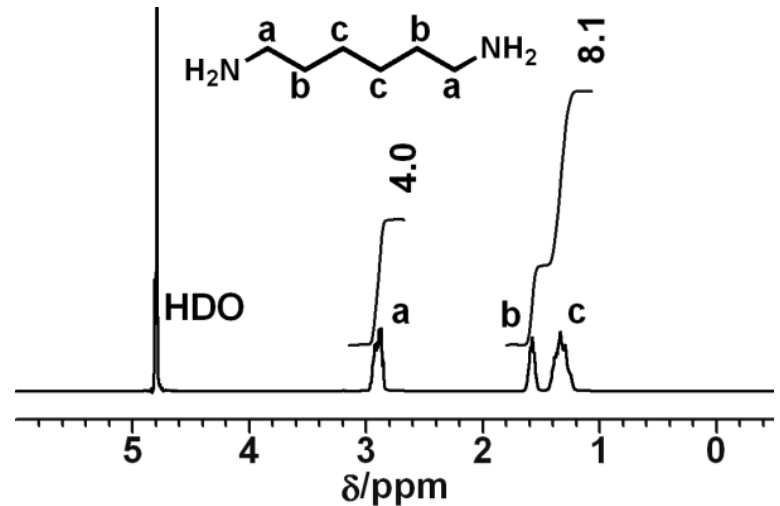


Figure 2

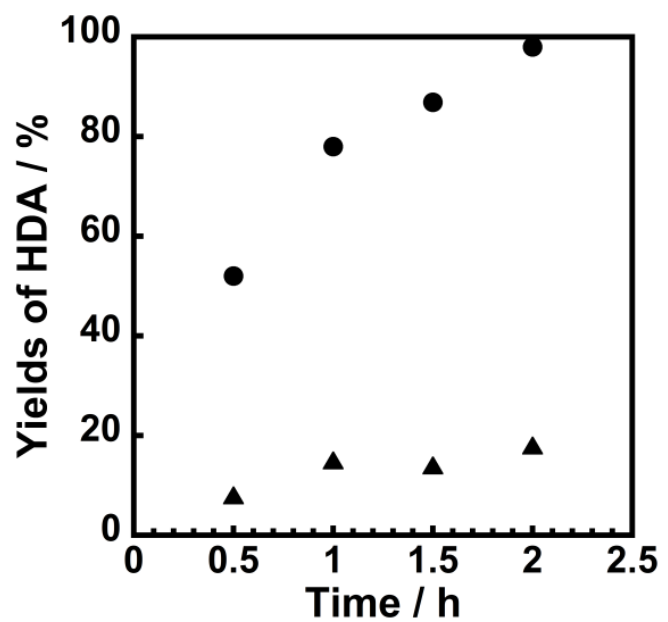


Figure 3

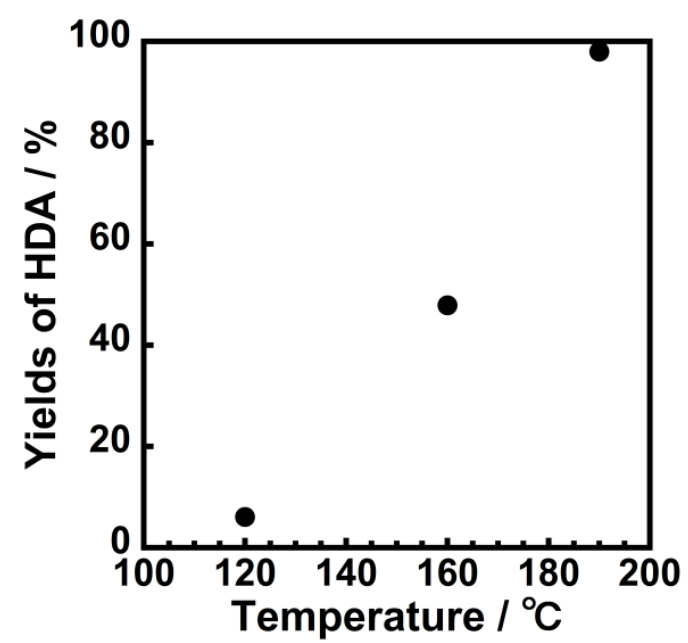


Figure 4

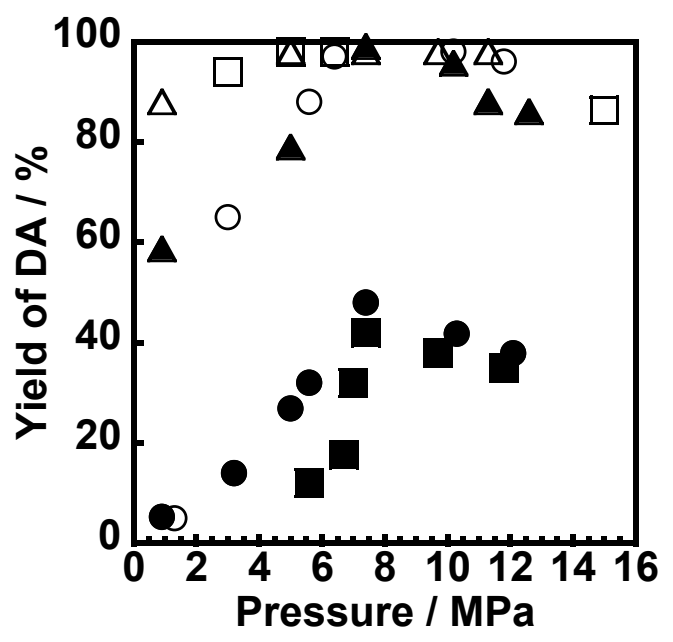


Figure 5

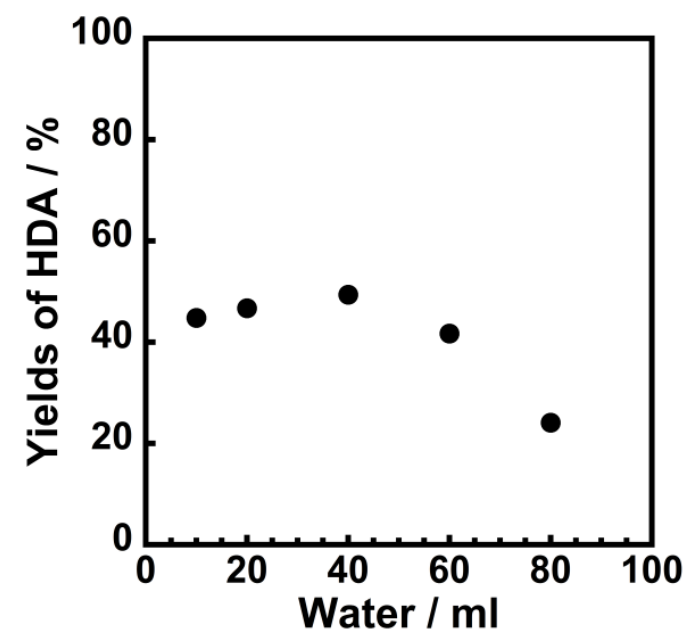


Figure 6

$\mathrm{H}_{2} \mathrm{O}+\mathrm{CO}_{2} \rightleftarrows \mathrm{H}_{2} \mathrm{CHO}_{3} \rightleftarrows \mathrm{H}^{+}+\mathrm{HCO}_{3}^{-} \rightleftarrows 2 \mathrm{H}^{+}+\mathrm{CO}_{3}^{2-}$

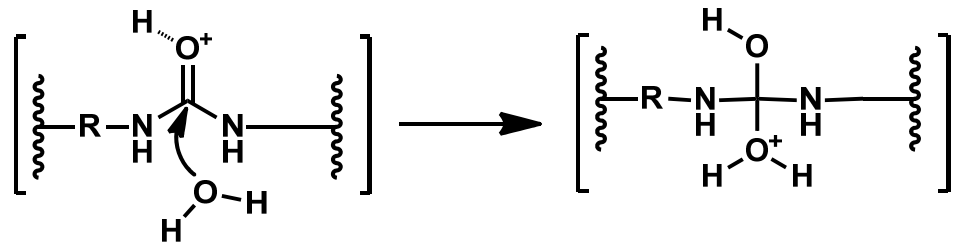

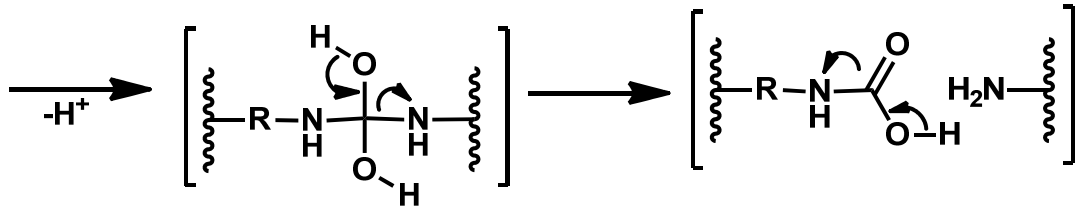

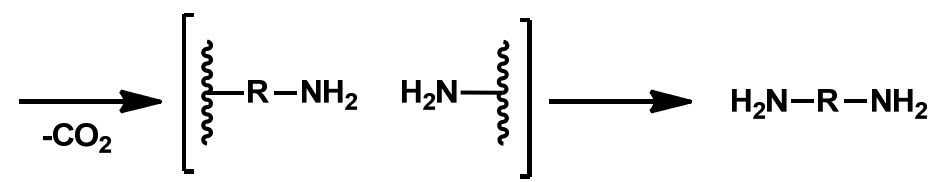


Captions

Figure 1. ${ }^{1} \mathrm{H}-\mathrm{NMR}$ spectrum of residue of filtrate (in $\mathrm{D}_{2} \mathrm{O}$ ).

Figure 2. The relationships between yield of HDA and reaction time at $190{ }^{\circ} \mathrm{C}$ under 7.0 MPa of $\mathrm{CO}_{2}(\bullet)$ and $7.0 \mathrm{MPa}$ of $\mathrm{N}_{2}(\boldsymbol{\Delta})$.

Figure 3. The relationships between yield of HDA and reaction temperature under 7.0 $\mathrm{MPa}$ of $\mathrm{CO}_{2}$, for $2 \mathrm{~h}$.

Figure 4. The relationships between yields of DAs and pressure of $\mathrm{CO}_{2}$ at $160{ }^{\circ} \mathrm{C}$ (filled symbols: ๑;HDA, $\mathbf{A}$;TDA and $\mathbf{\square}$;MDA) and $190{ }^{\circ} \mathrm{C}$ (open symbols: $\mathrm{O}$;HDA, $\triangle$;TDA and $\square$;MDA) under high pressure of $\mathrm{CO}_{2}$.

Figure 5. The relationships between yield of solid and amount of water at $160{ }^{\circ} \mathrm{C}$, for 2 h, under 7.0 MPa of $\mathrm{CO}_{2}$.

Figure 6. Mechanism of hydrolysis reaction of PUA. 